\title{
Diabetes in Older People: Comprehensive Approach
}

\author{
Óscar Moreno-Pérez ${ }^{1 *}$, Cristina Tejera ${ }^{2}$ and Rebeca Reyes-García ${ }^{3}$ \\ ${ }^{1}$ Endocrinology and Nutrition department, Alicante General University Hospital - Alicante Institute of Health and Biomedical Research \\ (ISABIAL), Alicante, Clinical Medicine department, Miguel Hernández University, Elche, Spain
}

${ }^{2}$ Endocrinology and Nutrition department, Complejo Hospitalario Universitario de Ferrol, Spain

${ }^{3}$ Endocrinology and Nutrition department, Torrecárdenas University Hospital, Almería, Spain

*Corresponding author: Óscar Moreno-Pérez, Endocrinology and Nutrition department, Alicante General University Hospital Alicante Institute of Health and Biomedical Research (ISABIAL), Alicante, Clinical Medicine department, Miguel Hernández University, Elche, Spain

\begin{abstract}
The clinical management of older people with diabetes requires a comprehensive evaluation and a holistic approach for the individualization of objectives and strategies of treatment. In older people with diabetes, geriatric syndromes, frailty and sarcopenia are considered at present as a third category of chronic complications. These situations are added to traditional microvascular and macrovascular complications, leading to significant disability and increasing the costs. In this context, two clinical scenarios can be considered: the first one, elderly subjects without significant comorbidities and good functional condition, in which an approach to diabetes similar to that of younger patients must be made. The second scenario, elderly and frail subjects, in which it will be essential a correct identification of these conditions and the evaluation of geriatric syndromes. This evaluation will guide the adaptation in the goals of treatment and in global management of diabetes.

Some basic principles should guide our decision-making: starting drugs at low - medium doses, with progressive increase according to tolerability; selection of drugs according to evidence-based medicine (considering the limited evidence in this age group), favoring agents with the lowest risk of hypoglycemia, avoid polypharmacy. Finally, patient's safety and quality of life should be the main objectives.
\end{abstract}

Keywords: Diabetes; Older; Frailty; Evidence-Based-Medicine

\section{Introduction}

Clinical management of older diabetes people requires a comprehensive evaluation and a holistic approach for the individualization of objectives and strategies of treatment. Geriatric syndromes, frailty and sarcopenia are considered at present as a third category of chronic complications [1]. These situations are added to traditional microvascular and macrovascular complications, leading to significant disability and a significant increase in costs.

In this context, two clinical scenarios can be considered: first, elderly subjects without significant comorbidities and without frailty, in which an approach to diabetes similar to that of younger patients must be made. The second scenario, elderly and frail subjects, in which a correct identification of frailty and an evaluation of geriatric syndromes is mandatory, guiding modifications in the goals of treatment and in the global management of diabetes.

\section{Initial Approach}

1. Consider evaluation of medical, functional (self-care skills) and geriatric sphere to establish a frame of reference that determines the objectives and therapeutic strategies diabetes management (Evidence B) [2].

2. Assess presence of geriatric syndromes (polypharmacy, cognitive impairment, depression, urinary incontinence, falls, chronic pain) as conditions that interfere with patient's management of diabetes and reduce quality of life (Evidence B) $[2]$. 


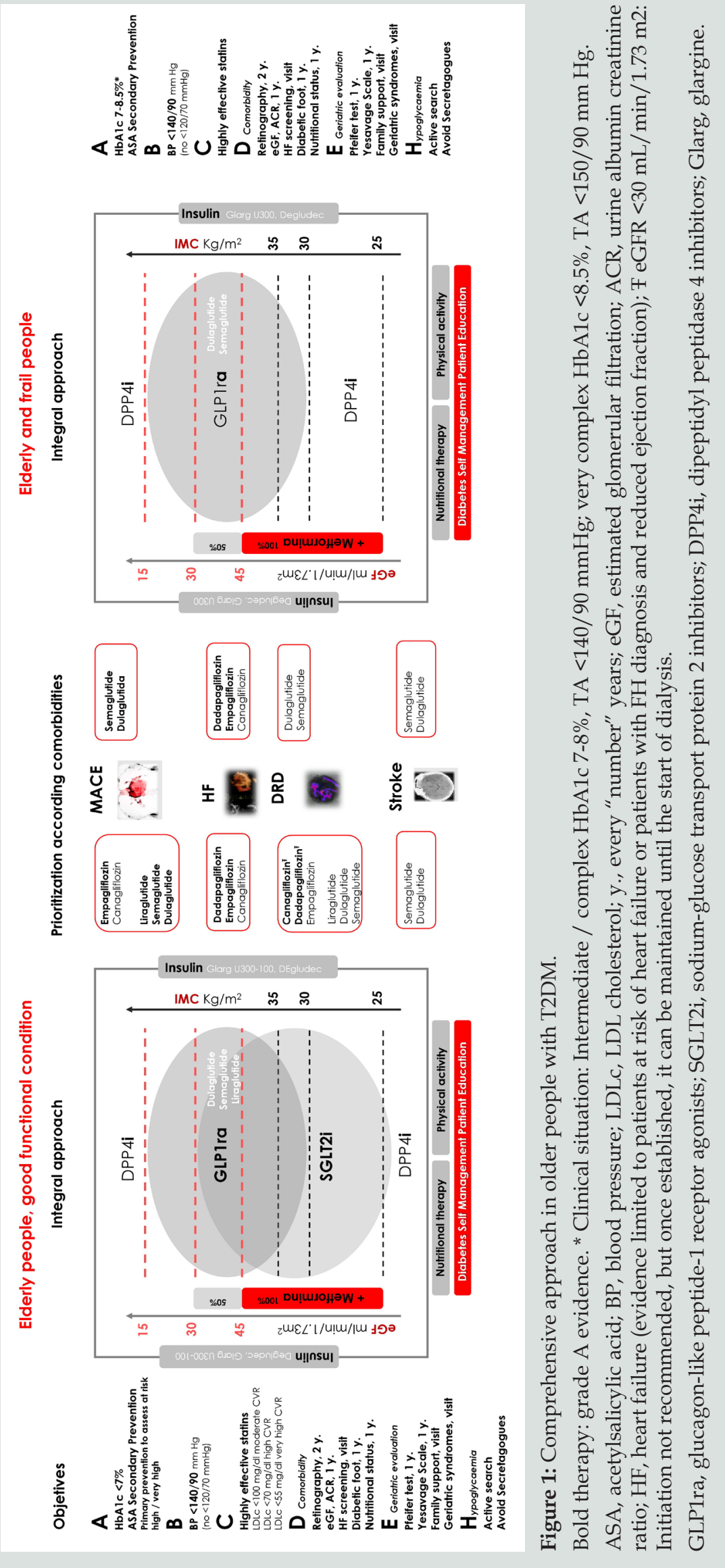


3. Evaluation of frailty. The most validated and simple evaluation tools are Fried criteria and FRAIL scale. Consider potentially reversible causes that contribute to frailty such as presence of hypothyroidism, vitamin D deficiency, anemia, etc., is advised [3].

4. In those over 65 years of age, an early diagnosis of mild cognitive alterations is recommended, at diagnosis and subsequently annually [2]. Pfeiffer questionnaire or Minimental test are validated tools. In patients with cognitive dysfunction, simplify treatment, and adapt care structure.

5. Patient safety, preferences and quality of life should be the main objectives.

Treatment objectives, therapeutic approach and the assessment of comorbidities, are shown in (Figure 1).

\section{Treatment Objectives (ABCDEH):}

\section{A. Glycemic control (A1c)}

General recommendation, which should always be individualized, is a target of HbA1c 7.5-8.5\% (58-69 mmol/l) in advanced frailty, and HbA1c 7-8\% (53-64 mmol/l) in mild to moderate frailty. In frailty subjects, $\mathrm{HbA} 1 \mathrm{c}<7 \%$ (53 mmol/l) should be avoided, especially if drugs with risk of hypoglycemia are used [2]. Many frail subjects have medical conditions that can interfere with HbA1c determination (chronic kidney disease, anemia, transfusions), and capillary blood glucose measurement may be necessary for assessing glycemic control [2].

\section{B. Blood pressure (BP)}

The objective of elderly subjects with diabetes, including those with dementia, is $<140 / 90 \mathrm{mmHg}$, avoiding values $<120 / 70 \mathrm{mmHg}$. A goal of $<150 / 90 \mathrm{mmHg}$ may be more suitable for the frail and dependent elderly. Whenever possible, measure BP standing and sitting, to detect orthostatic hypotension that increase the risk of falls. Withdrawal of treatments should be evaluated as frailty progresses [2,3].

\section{Hypercholesterolemia}

Statin treatment is recommended in the same situations as in non-elderly subjects: secondary prevention and primary prevention with high cardiovascular risk. Treatment of hypercholesterolemia in elderly patients has some differential characteristics. Lifestyle changes may not be possible. Furthermore, statin myopathy is more frequent (up to 10\%) due to sarcopenia, so it is advisable to use low or moderate doses of statins. Treatment of vitamin D deficiency can improve statin-associated myalgia [3]. In situations of advanced frailty and dependency, suspension of statins may be considered.

\section{Assessment of chronic complications}

It must be individualized, with particular attention to those with higher influence on functional state (retinopathy, diabetic macular edema and diabetic foot). Heart failure, chronic kidney disease, and vitamin B12 deficiency should not be forgotten $[2,4]$.

\section{E. Geriatric Evaluation}

Consider the assessment of geriatric syndromes: polypharmacy (use of three or five drugs simultaneously or the need to indicate one drug to supply the side effects of another), cognitive impairment, depression (Yesavage scale annually), urinary incontinence, falls, chronic pain (visual analogue pain scale), and frailty $[2,3]$.

\section{F. Hypoglycemia}

In older people prevention of hypoglycemia is especially important because of the repercussions on the risk of falls, fractures, and emergency department visits and hospitalization. Elderly patients have impaired recognition of hypoglycemia and difficulties in acquiring basic skills for self-care and for resolution of hypoglycemia, which determines a greater severity of the episodes. Also, there is a bi-directional relationship between hypoglycemia and cognitive decline [5].

\section{Comprehensive Pharmacological Treatment in the Elderly with T2DM}

In general terms, disease modifying therapies should be used in combination with metformin, that is, with benefit in morbidity - associated mortality, low risk of hypoglycemia, and benefits in terms of control of BP and excess of weight (if appropriate) [6].

The patient and their caregivers should be aware of the "sick days" rule for metformin and sodium-glucose transport protein 2 inhibitors (SGLT2i), to avoid the potential risk of impaired renal function, lactic acidosis, and euglycemic ketoacidosis. Also, simplification of complex regimens is recommended, especially in patients with insulin therapy, to reduce the risk of hypoglycemia and polypharmacy, always based on individualized HbA1c targets.

The use of SGLT2i in frail elderly patients with a diagnosis of heart failure (HF) with reduced ejection fraction (FEr), is a reasonable therapeutic option, given its potential benefits. Diuretic and blood pressure treatment must be revised to avoid volume depletion (hypotension, orthostatic hypotension, dizziness, syncope, and dehydration), and impaired kidney function.

DPP4 inhibitors (DPP4i) may be reserved for elderly people with renal function contraindicating other therapies, or those patients with normal weight, in whom the additional weight loss may be a problem; in this case, sitagliptin [7]. and linagliptin [8] must be prioritized. Sulfonylureas and glinides (hypoglycemia risk), and pioglitazone (risk of heart failure and fractures), must be avoided.

In frail elderly people with obesity, the use of weekly glucagonlike peptide-1 receptor agonists (GLP1ra) may be a good option 
given the low risk of hypoglycemia, the weight loss benefit, the potential benefits in comorbidities and the weekly administration [9]. Its use must be accompanied by adapted nutritional therapy, and appropriate physical activity recommendations (aerobic and resistance training) to avoid the loss of muscle mass, including strength, flexibility and balance exercises [10]. Also, the appearance of gastrointestinal effects should be monitored.

\section{References}

1. Wong E, Backholer K, Gearon E, Harding J, Freak-Poli R, et al. (2013) Diabetes and risk of physical disability in adults: a systematic review and meta-analysis. Lancet Diabetes Endocrinol 1(2): 106-114.

2. American Diabetes Association (2020) 12. Older Adults: Standards of Medical Care in Diabetes-2020. Diabetes Care 43(Supplement 1): S152-S162.

3. Alan Sinclair, Alison Gallagher (2019) Managing frailty and associated comorbidities in older adults with diabetes: Position Statement on behalf of the Association of British Clinical Diabetologists (ABCD) | ABCD (Diabetes Care) Pp: 1-17.

4. American Diabetes Association (2020) 4. Comprehensive Medical Evaluation and Assessment of Comorbidities: Standards of Medical Care in Diabetes-2020. Diabetes Care 43(Supplement 1): S37-S47.
5. Sheen Y-J, Sheu WHH (2016) Association between hypoglycemia and dementia in patients with type 2 diabetes. Diabetes Res Clin Pract 116 279-287.

6. Reyes-García R, Moreno-Pérez Ó, Tejera-Pérez C, Fernández-García D, Bellido-Castañeda V, et al. (2019) Document on a comprehensive approach to type 2 diabetes mellitus. Endocrinol Diabetes Nutr 66: 443458.

7. Bethel MA, Engel SS, Green JB, Huang Z, Josse RG, et al. (2017) Assessing the Safety of Sitagliptin in Older Participants in the Trial Evaluating Cardiovascular Outcomes with Sitagliptin (TECOS). Diabetes Care 40(4): 494-501.

8. McGuire DK, Alexander JH, Johansen OE, Perkovic V, Rosenstock J, et al. (2019) Linagliptin Effects on Heart Failure and Related Outcomes in Individuals with Type 2 Diabetes Mellitus at High Cardiovascular and Renal Risk in CARMELINA. Circulation 139(3): 351-61.

9. Valencia WM, Florez HJ, Palacio AM (2019) Suitable Use of Injectable Agents to Overcome Hypoglycemia Risk, Barriers, and Clinical Inertia in Community-Dwelling Older Adults with Type 2 Diabetes Mellitus. Drugs Aging 36(12):1083-1096.

10. Deutz NEP, Bauer JM, Barazzoni R, Biolo G, Boirie Y, et al. (2014) Protein intake and exercise for optimal muscle function with aging: recommendations from the ESPEN Expert Group. Clin Nutr 33(6): 929936.

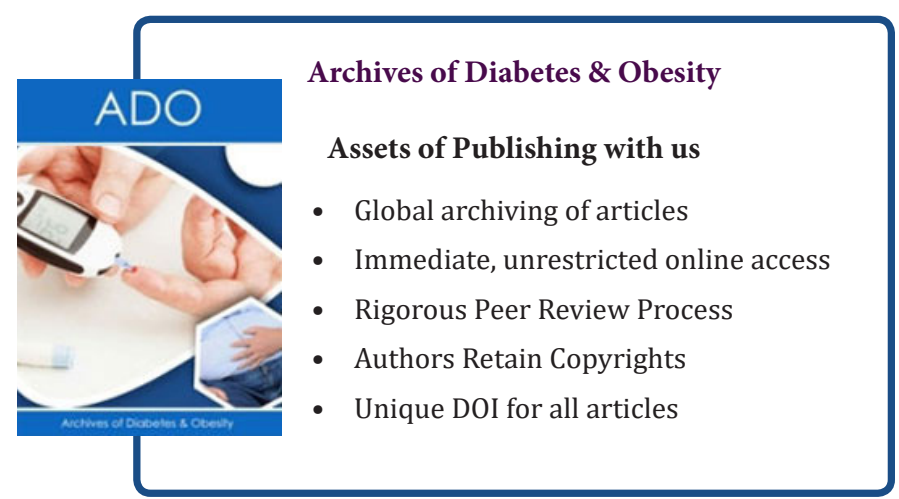

